\title{
Fixed Cutaneous Sporotrichosis Due to Sporothrix globosa
}

This article was published in the following Dove Press journal:

Clinical, Cosmetic and Investigational Dermatology

\author{
Fang Liu (iD \\ Yingmei Liu $^{2}$ \\ $\mathrm{Na}$ Yuan' \\ Xiuying Zhang' \\ Mei Cao' \\ Jie Dong' \\ Jinqing Zhang' \\ 'Department of Dermatology, Beijing \\ Chaoyang Hospital, Capital Medical \\ University, Beijing 100020, People's \\ Republic of China; ${ }^{2}$ Laboratory of Clinical \\ Microbiology and Infectious Diseases, \\ Department of Pulmonary and Critical \\ Care Medicine, China-Japan Friendship \\ Hospital, Beijing 100029, People's \\ Republic of China
}

Purpose: This report describes a case of a skin sporotrichosis infection and the steps taken to identify an effective antifungal treatment.

Patients and Methods: A 50-year-old woman from Jilin province, China, presented complaining of a small mass that had been on her right upper eyelid for two years. A skin biopsy was taken and submitted for bacterial and mycological assessment. Bacterial culture from the lesion was negative, but a fungal culture was positive. In vitro susceptibility test was performed to assess its susceptibility to antifungal drugs.

Results: The skin biopsy showed infectious granuloma. Fungal culture was identified as Sporothrix globosa based on both the morphological features and confirmation by the molecular method; it was resistant to many kinds of antifungal drugs, including amphotericin $\mathrm{B}$, voriconazole, fluconazole, and caspofungin. However, it was relatively sensitive to itraconazole. The patient was prescribed $0.2 \mathrm{~g}$ itraconazole to be taken twice per day. One month later, she had almost completely recovered from her symptoms. The treatment lasted for 3 months and her liver function and renal function were normal at the endpoint.

Conclusion: Itraconazole was an effective treatment in this case of a multidrug-resistant sporotrichosis caused by $S$. globosa.

Keywords: fixed cutaneous sporotrichosis, Sporothrix globosa

\section{Plain Language Summary}

Why was this study done? Sporotrichosis is an infection caused by the Sporothrix fungi. It usually begins with skin lesions but can slowly spread to other body parts and cause serious complications if left untreated. In China, the most common cause is Sporothrix globosa, which is difficult to treat with most antifungal drugs. Therefore, it is important to identify effective treatment. What did the researchers do? We biopsied a sample from a 50-year-old woman in China suffering from a mass that had been on her eyelid for 2 years. We found that she had a fungal infection. We then identified the fungal at species level based on both the morphological features and confirmation by the molecular method. Next, we tested various antifungal drugs to discover which drug has the best activity against this infection. What was learned? We found that the drug itraconazole was the most effective treatment of all those tested against S. globosa in this case. The patient's symptoms were cured after using itraconazole for 1 month.

\section{Introduction}

Sporothrix spp. are dimorphic fungi found in the soil and the causative agents of sporotrichosis. $^{1,2}$ They are found worldwide, especially in tropical/temperate regions. Sporotrichosis is a chronic infection of the skin and subcutaneous tissue and adjacent lymphatic vessels, characterized by nodular lesions that lead to
Department of Dermatology, Beijing

Chaoyang Hospital, Capital Medical

University, 8 Gongti South Road, Beijing

100020, People's Republic of China

Tel +86-18611369216

Email roseliufang@qq.com
Clinical, Cosmetic and Investigational Dermatology 2021:14 9|-96 
abscesses and ulcers. The pathogen enters the body via breaches in the skin barrier. ${ }^{3-5}$ If left untreated, the infection can spread to the joints, bones, muscles, central nervous system, lungs, or urogenital tract. Human disease due to Sporothrix has a broad range of clinical manifestations and can be classified into fixed cutaneous, lymphocutaneous, disseminated cutaneous, and extracutaneous sporotrichosis. The gold standard for the diagnosis of sporotrichosis is culturing; however, serologic, histopathologic, and molecular approaches have been recently adopted to diagnose this mycosis. ${ }^{6}$

In China, most patients are infected with Sporothrix globosa $($ S. globosa $),{ }^{7-9}$ a notably drug-resistant fungus. ${ }^{2,10}$

\section{Patients and Methods}

\section{Case}

A 50-year-old female patient from Jilin Province in China was admitted to our department on November 22, 2018. She complained of a small mass that had been present for the past 2 years. She stated that she had a burn from cooking oil on her right upper eyelid, which subsequently blistered, and a mass slowly grew in the area thereafter. One year ago, she was diagnosed with a fungal skin infection at a local hospital. She was administered $0.25 \mathrm{~g}$ terbinafine orally for approximately 1 year, and butenafine cream was used topically. However, the skin lesion often discharged purulent secretions, leading her to seek further treatment. She was otherwise healthy, and her immunological state was normal, with no history of drug or food allergies. Dermatological examination showed a small mass on the right upper eyelid with rough keratinization. It was hard and small, exudates were observed after compressions, and no other eruption or rash was found throughout the body.
Skin biopsy specimens were scraped from the right upper eyelid lesion, cultured on Sabouraud's dextrose agar (SDA), and incubated at both $27^{\circ} \mathrm{C}$ and $37^{\circ} \mathrm{C}$. Initially, white, smooth, and membranous colonies were observed after 3 days of incubation at $27^{\circ} \mathrm{C}$; later, they turned brown, with a central depression and a surrounding uplift, and were wrinkled and waxy, with folded surfaces after 8 days of incubation (Figure 1A and B). The colonies grew very slowly at $37^{\circ} \mathrm{C}$ and were small, white, and yeast-like (Figure 1C) after 8 days. Microscopic examination after lactophenol cotton-blue staining of the culture revealed mycelia with slender and tapered tips. Conidiophores arose at right angles from thin septate hyphae, erect and tapered toward the apex. Conidia were hyaline or slightly pigmented and obovoid or pear-shaped. These conidia were formed in clusters on tiny denticles via sympodial proliferation at the conidiophore apex, giving the typical "flower" image (Figure 2A and B). Fungal culture was identified being based on the morphological features and confirmed via the molecular sequencing of D1/D2, $\beta$-tubulin and internal transcribed spacer (ITS) region genes. ${ }^{11-14}$ The primer sequences used for polymerase chain reaction (PCR) are shown in Table 1. The sequences obtained from PCR (see supplementary material) were checked by BLAST in NCBI dataset. Phylogenetic trees based on sequence data from 28S rDNA D1/D2 region. The phylogenetic analysis was performed with the neighbor-joining method, calculated using the p-distance model. Values on each branch indicate the occurrence (\%) of the branching order in 1000 bootstrapped trees. Ceratocystiopsis ranaculosa CBS 216.88 was used as an outgroup. Multiple sequence alignments were performed using Clustal (version X2) software and checked by BLAST in NCBI dataset. Phylogenetic trees were generated from MEGA (version 6.0) software.

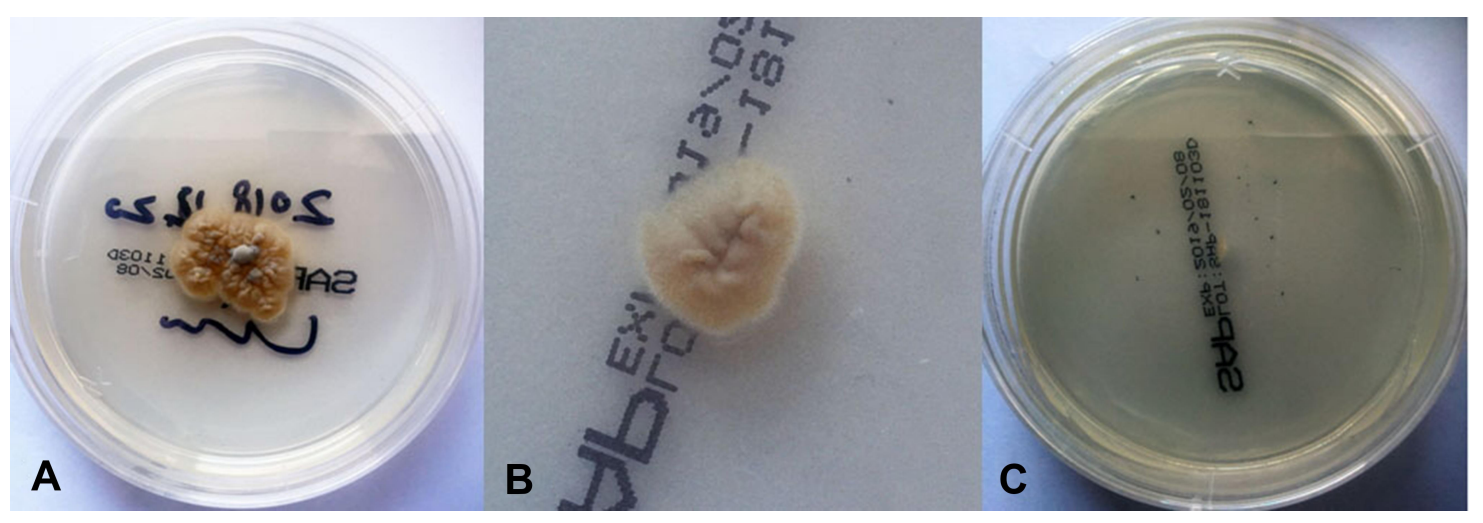

Figure I Tissue culture of skin lesion scrapes in SDA medium at $27^{\circ} \mathrm{C}$. After 8 days, colonies appeared waxy and light brown, with a central depression and a peripheral uplift (A and B). Tissue culture of patient's skin scrapes in SDA medium at $37^{\circ} \mathrm{C}$ for 8 days. Colonies grew very slowly and appeared small, white, and yeast-like (C). 

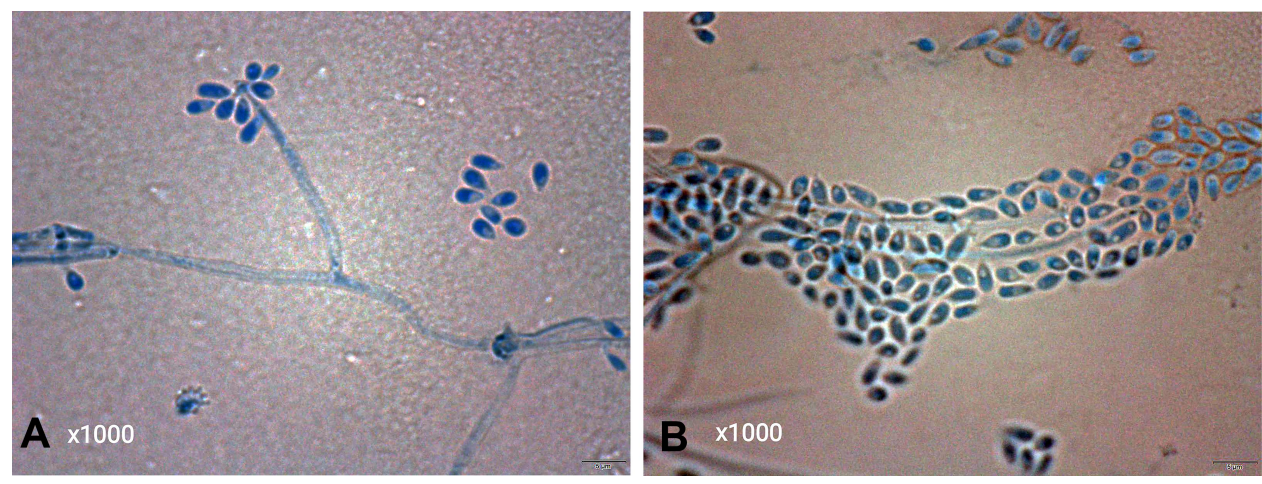

Figure 2 Colonies stained with lactophenol cotton blue dye after tableting and coating and subsequently observed under a microscope (I000x magnification). Clear and divided mycelia could be observed with slender, branched, and tapered tips (A). Conidium stalks appeared pear-shaped and were clustered similarly to flowers (B). Conidium was arranged conically along the axis.

In vitro antifungal susceptibility tests with fluconazole, itraconazole, voriconazole caspofungin, and amphotericin B were performed using the E-test method as previously described. ${ }^{15}$

\section{Biopsy}

We biopsied the skin lesion and found an infectious granuloma (Figure 3A, 40× magnification) with various infiltrating cells, including neutrophils, lymphocytes, epithelioid cells, monocytes (Figure 3B, 100× magnification), plasmid cells (Figure 3C, 400× magnification), and macrophages in the dermis by hematoxylin and eosin staining (Figure 3D, 400× magnification). No hyphae or asteroid bodies were observed even with periodic acidSchiff staining.

\section{Results}

Phylogenetic tree was generated and showed that our identified strain named as CY20191101 was $100 \%$ of the homology to Sporothrix globosa (S. globosa) CBS129719 and CBS 129718 (Figure 4). Hence, the strain was identified as $S$. globosa. A proven skin sporotrichosis infection caused by $S$. globosa was confirmed.
Drug-sensitivity tests indicated high minimal inhibitory concentrations (MICs) for common antifungal agents (256 $\mathrm{mg} / \mathrm{L}$ for fluconazole, $32 \mathrm{mg} / \mathrm{L}$ for voriconazole, caspofungin, and amphotericin B), while MICs were relatively lower for itraconazole $(0.75 \mathrm{mg} / \mathrm{L})$. Therefore, the patient was prescribed $0.2 \mathrm{~g}$ itraconazole twice daily. Four weeks later, she was almost cured (Figure 5). The treatment lasted for three months, and liver and renal functions were normal at the endpoint. (ALT $28 \mathrm{U} / \mathrm{L}$, AST $30 \mathrm{U} / \mathrm{L}$, Bun $4.1 \mathrm{mmol} / \mathrm{L}, \mathrm{Cr} 66 \mathrm{umol} / \mathrm{L}$ )

\section{Discussion}

Sporotrichosis is a chronic, granulomatous, subcutaneous infection caused by dimorphic members of the genus Sporothrix, including at least six sibling phylogenetic species: S. pallida, S. brasiliensis, S. globosa, S. luriei, S. mexicana, and S. schenckii. ${ }^{16}$ In China, S. globosa is predominant, ${ }^{7-9,11}$ except for in the Nanchang area, where S. schenckii occurs along with S. globosa. ${ }^{16}$

This patient was from Jilin province where is in the northeast of China, and sporotrichosis is the most common deep cutaneous mycosis in that area. The skin lesion was limited to her right upper eyelid, and S. globosa was

Table I Primer Sequences Used for PCR Amplification

\begin{tabular}{|l|l|l|}
\hline Primers & Targeted Region & Primer Sequences \\
\hline $\begin{array}{l}\text { ITSI } \\
\text { ITS4 }\end{array}$ & Internal transcribed spacer regions & $\begin{array}{l}\text { 5'-TCCGTAGGTGAACCTGCGG-3' } \\
\text { 5'-TCCTCCGCTTATTGATATGC-3' }\end{array}$ \\
\hline $\begin{array}{l}\text { NL-I } \\
\text { NL-4 }\end{array}$ & DI/D2 variable domains of the 28S rDNA gene & $\begin{array}{l}\text { 5'-GCATATCAATAAGCGGAGGAAAAG-3' } \\
\text { 5'-GGTCCGTGTTTCAAGACG-3' }\end{array}$ \\
\hline $\begin{array}{l}\text { bt2a: } \\
\text { bt2b: }\end{array}$ & Beta-tubulin & $\begin{array}{l}\text { 5'-GGTAACCAAATCGGTGCTGCTTTC-3' } \\
\text { 5'-ACCCTCAGTGTAGTGACCCTTGGC-3' }\end{array}$ \\
\hline
\end{tabular}



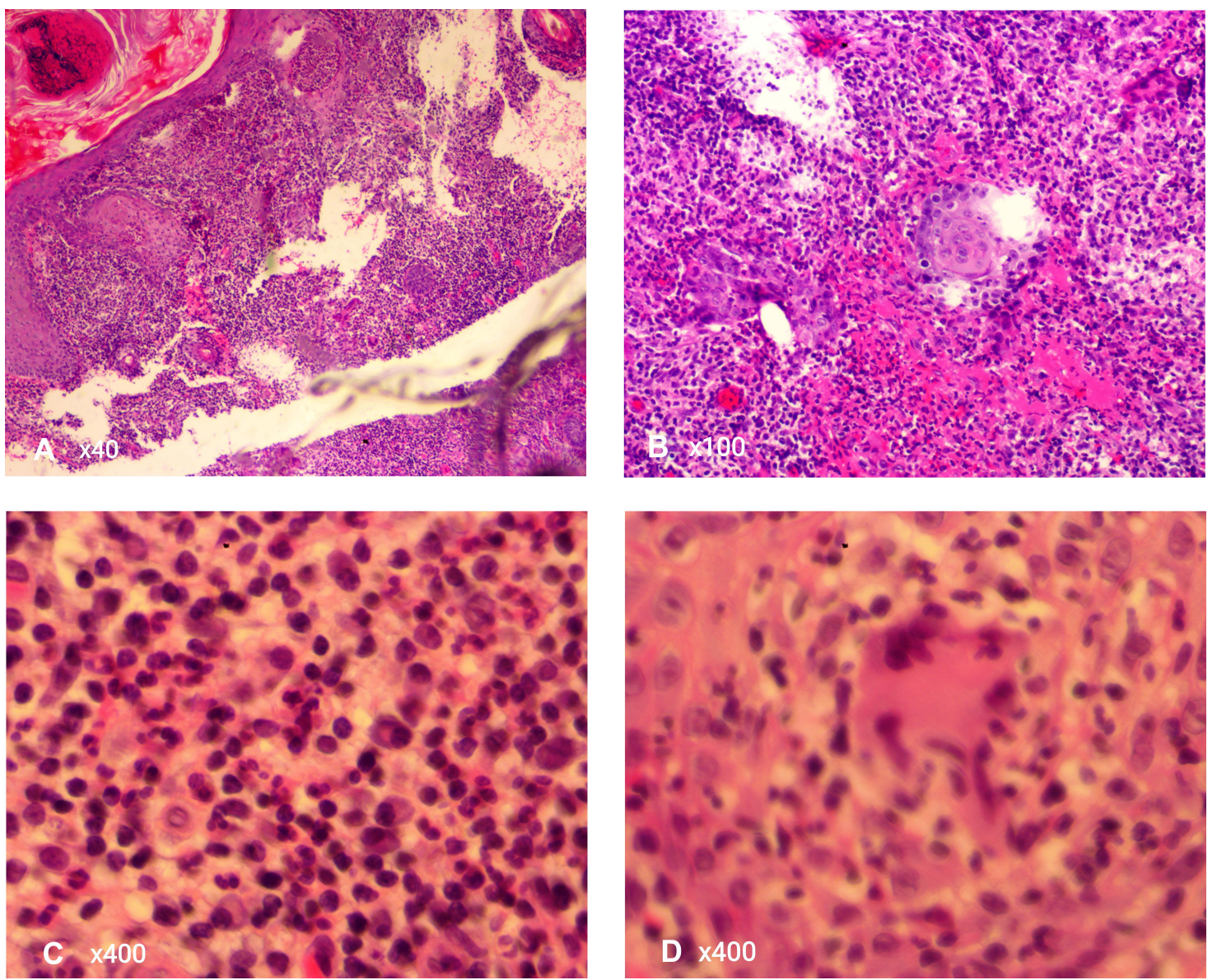

Figure 3 Pathological manifestation showed an infectious granuloma with various infiltrating cells in the dermis (A and B), including neutrophils, lymphocytes, plasmid cells, (C) epithelioid cells, and monocytes and macrophages (D). No hyphae or satellite bodies were observed.

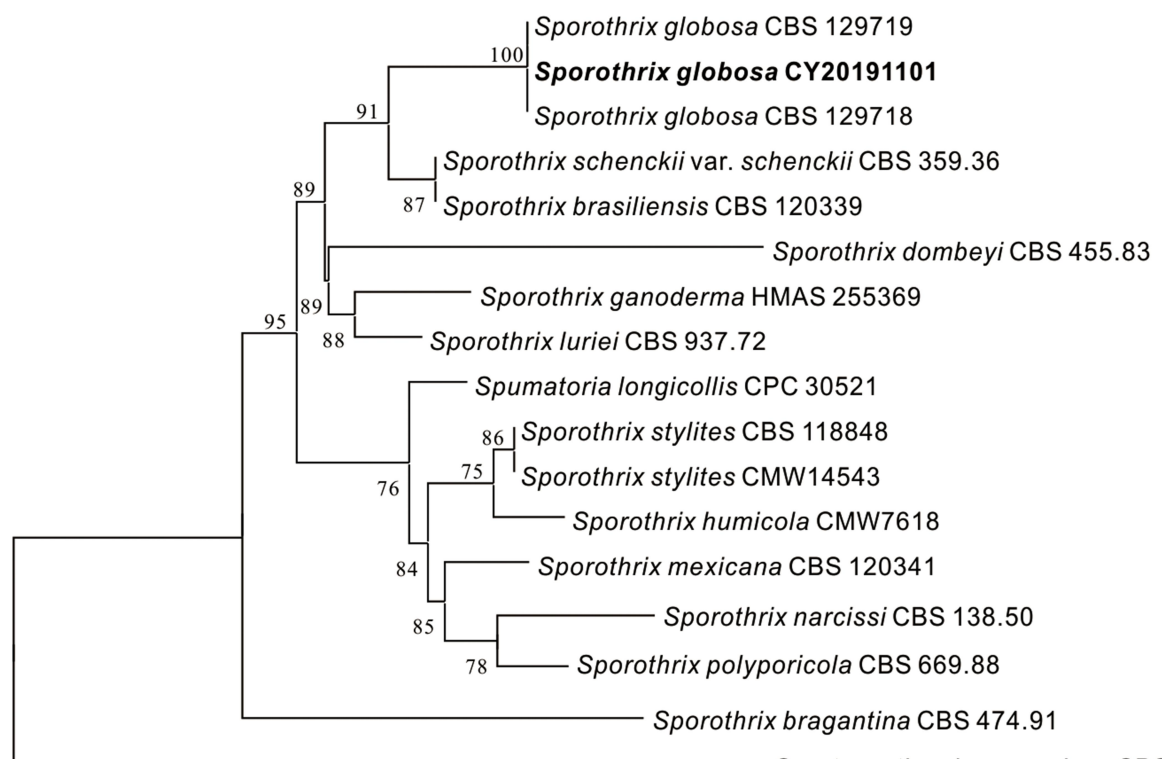

Ceratocystiopsis ranaculosa CBS 216.88

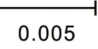

Figure 4 Phylogenetic tree showed that our identified strain named as CY20I9II0I was 100\% of the homology to Sporothrix globosa CBSI297I9 and CBS I297I8. 

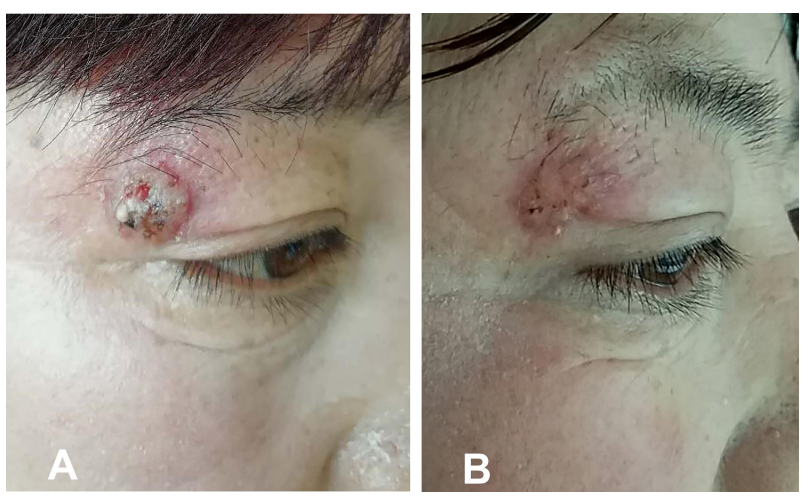

Figure 5 Patient's condition before and after I month of treatment. The patient (A) was prescribed $0.2 \mathrm{~g}$ itraconazole twice daily for 3 months. The lesion improved significantly after four weeks of treatment (B).

isolated from the lesion culture, so the clinical diagnosis was fixed cutaneous sporotrichosis.

In most sporotrichosis cases, histopathological detection of the etiologic agent is difficult due to a low fungal load, with abundant cells being occasionally found in tissues only. ${ }^{16}$ For this patient, we were unable to identify the fungal cells on the histological slides.

Because this patient was administered terbinafine for 1 year before she came to our clinic, we performed drugsensitivity testing to ascertain drug resistance. Drug sensitivity tests suggested that the strain was resistant to several antifungal drugs but was sensitive to itraconazole. According to the guidelines for treating sporotrichosis, itraconazole should be taken for 3-6 months. ${ }^{7,17}$ This patient took itraconazole for 4 weeks before satisfactory results were seen. The treatment lasted for three months, she did not complain of any discomfort. Follow-up visits were performed every month for 6 months, and no relapse has been observed to date.

\section{Conclusion}

Sporotrichosis is a comparatively rare skin disease caused by the Sporothrix spp. However, if left untreated, it can spread to other organs and cause serious complications. The case of a fixed cutaneous sporotrichosis from China caused by S. globosa presented here shows the need for both rapid and improved diagnostics for fungal diseases and drug sensitivity testing to determine the most effective course of treatment. Drug sensitivity tests are recommended whenever possible. E-test was easily performed upon our experiment.

\section{Ethics Statement}

The procedures and medications used in this study and the publication of the case details were approved by the Ethics
Committee of Beijing Chaoyang Hospital, Capital Medical University. Written informed consent for publication was obtained from the patient. The written informed consent also included permission for the publication of case details and accompanying images.

\section{Acknowledgments}

This study was supported by the Scientific Research Foundation for Returned Scholars of the Department of Human Resources and Social security of Beijing City.

\section{Disclosure}

The authors report no conflicts of interest in this work.

\section{References}

1. Chakrabarti A, Bonifaz A, Gutierrez-Galhardo MC, et al. Global epidemiology of sporotrichosis. Med Mycol. 2015;53:3-14. doi:10.1093/mmy/myu062

2. Orofino-Costa R, Macedo PM, Rodrigues AM, et al. Sporotrichosis: an update on epidemiology, etiopathogenesis, laboratory and clinical therapeutics. An Bras Dermatol. 2017;92:606-620. doi:10.1590/ abd1806-4841.2017279

3. da Silva RF, Bonfitto M, da Silva Junior FIM, et al. Sporotrichosis in a liver transplant patient: a case report and literature review. Med Mycol Case Rep. 2017;17:25-27. doi:10.1016/j.mmcr.2017.06.005

4. Gremião IDF, de Miranda LHM, Reis EG, et al. Zoonotic epidemic of sporotrichosis: cat to human transmission. PLoS Pathog. 2017;13: e1006077. doi:10.1371/journal.ppat.1006077

5. Eaton AE, Ahmad S. Sporotrichosis: when uncommon etiology makes a treatable disease nearly fatal. $S$ D Med. 2018;71:512-513.

6. Oliveira MM, Almeida-Paes R, Gutierrez-Galhardo MC, et al. Molecular identification of the Sporothrix schenckii complex. Rev Iberoam Micol. 2014;31(1):2-6. doi:10.1016/j.riam.2013.09.008

7. Mycology Group of Chinese Society of Dermatology, Subcommittee on Medical Mycology; Chinese Dermatologist Association, Mycology Group; Committee on Dermatology and Venereology, China Society of Integrated Traditional Chinese and Western Medicine. Guidelines for the diagnosis and treatment of sporotrichosis. Chin J Dermatol. 2016;49:456-459.

8. Zhang Y, Hagen F, Stielow B, et al. Phylogeography and evolutionary patterns in Sporothrix spanning more than 14000 human and animal case reports. Persoonia. 2015;35:1-20. doi:10.3767/003158515X 687416

9. Yu X, Wan Z, Zhang Z, et al. Phenotypic and molecular identification of Sporothrix isolates of clinical origin in Northeast China. Mycopathologia. 2013;176(1-2):67-74. doi:10.1007/s11046-013-9668-6

10. Rodrigues AM, de Hoog GS, de Cássia Pires D, et al. Genetic diversity and antifungal susceptibility profiles in causative agents of sporotrichosis. BMC Infect Dis. 2014;14:219. doi:10.1186/14712334-14-219

11. Liu TT, Zhang K, Zhou X. Molecular identification of Sporothrix clinical isolates in China. $J$ Zhejiang Univ Sci B. 2014;15 (1):100-108. doi:10.1631/jzus.B1300136

12. Sasaki AA, Fernandes GF, Rodrigues AM, et al. Chromosomal polymorphism in the Sporothrix schenckii complex. PLoS One. 2014;9 (1):e86819. doi:10.1371/journal.pone.0086819

13. Rudramurthy SM, Shankarnarayan SA, Hemashetter BM, et al. Phenotypic and molecular characterisation of Sporothrix globosa of diverse origin from India [published online ahead of print, $2020 \mathrm{Jul} 30$ ]. Braz J Microbiol. 2020. doi:10.1007/s42770-020-00346-6 
14. Flórez-Muñoz SV, Alzate JF, Mesa-Arango AC. Molecular identification and antifungal susceptibility of clinical isolates of sporothrix schenckii complex in Medellin, Colombia. Mycopathologia. 2019;184(1):53-63. doi:10.1007/s11046-018-0310-5

15. Gutierrez-Galhardo MC, Zancopé-Oliveira RM, Monzón A, et al. Antifungal susceptibility profile in vitro of Sporothrix schenckii in two growth phases and by two methods: microdilution and E-test. Mycoses. 2010;53(3):227-231. doi:10.1111/j.1439-0507.2009.01701.x
16. Gu A, Zhang X, Ma F, et al. Fixed cutaneous sporotrichosis in a patient with numerous fungal elements. Med Mycol Case Rep. 2020;29:32-34. doi:10.1016/j.mmcr.2020.06.001

17. Kauffman CA, Bustamante B, Chapman SW, et al. Infectious diseases society of America. Clinical practice guidelines for the management of sporotrichosis: 2007 update by the infectious diseases society of America. Clin Infect Dis. 2007;45(10):1255-1265. doi:10.1086/ 522765

\section{Publish your work in this journal}

Clinical, Cosmetic and Investigational Dermatology is an international, peer-reviewed, open access, online journal that focuses on the latest clinical and experimental research in all aspects of skin disease and cosmetic interventions. This journal is indexed on CAS.
The manuscript management system is completely online and includes a very quick and fair peer-review system, which is all easy to use. Visit http://www.dovepress.com/testimonials.php to read real quotes from published authors. 